\title{
Observer variability in histopathological reporting of non-small cell lung carcinoma on bronchial biopsy specimens
}

R A Burnett, S R Howatson, S Lang, F D Lee, A M Lessells, K M McLaren, E R Nairn, S Ogston, A J Robertson, J G Simpson, G D Smith, H B Tavadia, F Walker

\begin{abstract}
Aims-To evaluate the ability of histopathologists to sub-classify non-small cell lung carcinomas on bronchial biopsy material using the current World Health Organisation (WHO) classification.

Methods-Twelve histopathologists each reviewed 100 randomly selected bronchial biopsy specimens which had originally been reported as showing non-small cell lung carcinoma. For each case, two sections were circulated, one stained by haematoxylin and eosin and the other by a standard method for mucin (alcian bluel periodic acid Schiff). The participants were allowed to indicate their degree of confidence in their classification of each case. A standard proforma was completed and the results were analysed using $\kappa$ statistics.
\end{abstract}

Results-Where the participants were confident in their classification, they were actually quite good at sub-classifying the non-small cell carcinoma sections $(\kappa=$ $0 \cdot 71$, standard error $=0.058$ ). Overall, however, the results were only fair $(\kappa=$ $0 \cdot 39$, standard error $=0.034$ ).

Conclusions-The majority of non-small cell lung carcinomas can be correctly categorised on adequate bronchial biopsy material. Where a confident diagnosis was made, both squamous carcinoma $(\kappa=0 \cdot 73)$ and adenocarcinoma $(\kappa=0 \cdot 83)$ were well recognised.

(F Clin Pathol 1996;49:130-133)

Keywords: histopathology audit, lung cancer, non-small cell carcinoma.

The University of Glasgow Department of Pathology, The Western Infirmary, Glasgow G11 6NT R A Burnett

S R Howatson

$S$ Lang

F D Lee

A M Lessells

K M McLaren

E R Nairn

$S$ Ogston

A J Robertson

J G Simpson

G D Smith

H B Tavadia

F Walker

Correspondence to: Dr R A Burnett.

Accepted for publication 18 October 1995

It falls to the histopathologist or cytopathologist to provide the "tissue diagnosis" of bronchial or lung carcinoma. Most histopathologists use a classification based on the World Health Organisation's (WHO) second classification (1981) (table 1). ${ }^{1}$ In a previous paper in this Journal $^{2}$ we demonstrated that using bronchial biopsy material histopathologists are extremely accurate in the clinically important categorisation of lung cancer into small cell and non-small cell types but sub-classification of non-small cell carcinoma was performed much less well. The previous study was, however, designed to answer the important question of our ability to sub-divide lung carcinomas into small cell and non-small cell types and as a mucin stain was not circulated, it was not surprising that the sub-classification of nonsmall cell tumours was performed less well. Some investigators have asserted ${ }^{3}$ that the subcategorisation of non-small cell tumours should not be attempted on this type of material. However, we decided that we would attempt this sub-classification in a second study designed more specifically for that purpose.

The tissue diagnosis of lung cancer is usually made on material obtained by bronchial biopsy or by the cytological examination of sputum or bronchial washings or brushings. In this second study we again decided to review bronchial biopsy material in an attempt to evaluate the degree of confidence which can be given to the histological sub-classification of non-small cell lung cancer by non-specialist histopathologists, thereby testing the "robustness" of the existing terminology and classification when applied to this type of specimen.

The WHO classification ${ }^{1}$ is based upon tumour differentiation as seen in routinely produced sections examined by light microscopy and it does not take into account any additional evidence of differentiation which may become apparent using other more specialised techniques, such as immunohistochemistry or electron microscopy. It has also long been recognised that lung cancers are notoriously

Table 1 World Health Organisation histological classification of lung tumours (1981)

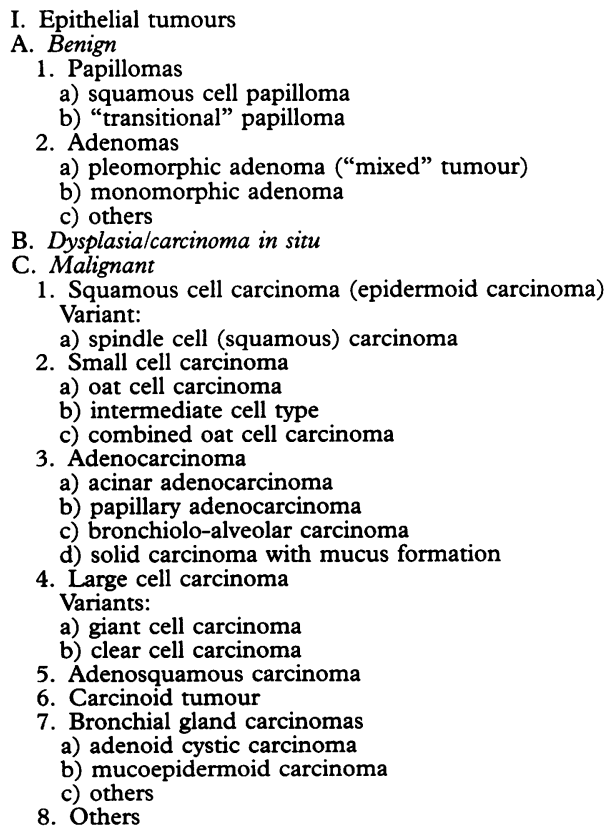

Squamous cell carcinoma (epidermoid carcinoma)

Variant:

a) spindle cell (squamous) carcinoma

2. Small cell carcinoma

a) oat cell carcinoma

b) intermediate cell type

combined oat cell carcinoma

3. Adenocarcinoma

a) acinar adenocarcinoma

b) papillary adenocarcinoma

bronchiolo-alveolar carcinoma

d) solid carcinoma with mucus formation

4. Large cell carcinoma

Variants:

a) giant cell carcinoma

b) clear cell carcinoma

5. Adenosquamous carcinoma

6. Carcinoid tumour

7. Bronchial gland carcinomas

a) adenoid cystic carcinoma

b) mucoepidermoid carcinoma

c) others

8. Others 
BRONCHIAL BIOPSY PATHOLOGY ॥

Non-small cell circulation

SLIDE NO.

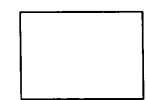

(Please put tick in appropriate boxes)

A) Small cell carcinoma

B) Non-small cell carcinoma

If non-small cell carcinoma is it:

1) squamous

2) adenocarcinoma

3) carcinoma NOS (ie neither 1 nor 2)

C) Not carcinoma

Re: Diagnosis

Very high level of confidence

Some doubt as to precise category

Pathologist Code: $\square$

Simple proforma completed for each section studied.

heterogeneous in their differentiation, different areas of the same tumour expressing completely different phenotypes. ${ }^{45}$ In biopsy specimens only a tiny amount of tumour is present and in practice more than one differentiation pattern is rarely seen. We therefore decided that the examination of a single haematoxylin and eosin stained section together with a section stained for mucin from each case would give a reasonable indication of our ability to assess tumour differentiation.

\section{Methods}

Twelve histopathologists took part in this study. As in our previous studies, ${ }^{267}$ they had been chosen to represent Scottish histopathology as a whole. Only one (RAB) had any special expertise in respiratory pathology. The panel members came from pathology laboratories in Aberdeen $(n=2)$, Dundee $(n=2)$, Edinburgh $(n=2)$, Airdrie $(n=1)$, Stirling $(n=1)$, Ayrshire $(n=1)$, and Glasgow $(n=3)$. All of the participants were of consultant grade but they varied in experience (five to 30 years) and the nature of their substantive post (University staff $=4$, NHS staff $=8$ ). The statistical analysis
Table 2 Subclassification of non-small cell carcinoma (all cases)

\begin{tabular}{lll}
\hline Type & $\kappa$ value & $\begin{array}{l}\text { Value in } \\
\text { previous work }\end{array}$ \\
\hline Squamous cell & $0 \cdot 44$ & $(0 \cdot 30)$ \\
Adenocarcinoma & 0.54 & $(0 \cdot 28)$ \\
Large cell undifferentiated & 0.25 & $(0 \cdot 17)$ \\
Overall & 0.39 & $(0 \cdot 25)$ \\
\hline
\end{tabular}

was performed by a senior medical statistician (SO) from the Department of Epidemiology and Public Health of the University of Dundee.

Ten of the 12 participants were asked to extract 10 consecutive bronchial biopsy specimens which had been originally reported as showing non-small cell lung cancer from the files of their respective laboratories, starting from an arbitrary date of their own choosing. Carcinoid tumours and small cell carcinomas were specifically excluded. Two sections were produced from each case, one was stained by haematoxylin and eosin and the other by a standard alcian blue/periodic acid Schiff (PAS) method. The 100 cases were then randomly allocated and re-numbered. The sections were circulated in batches round the 12 histopathologists who then completed a simple proforma (figure) for each section examined. The completed proformas were collated centrally.

The main problem with the analysis of studies of this kind is the lack of knowledge of the "correct" diagnosis for each slide. In some studies an "expert peer group" diagnosis has been used. In this study, as in some of our previous work, ${ }^{267} \mathrm{~K}$ statistics were used to analyse the results. Kappa statistics are a measure of overall agreement and do not require any assumption about the "correct" diagnosis. They include a correction for the amount of agreement which would be expected by chance alone. The value of $\kappa$ can range from -1.0 to $+1 \cdot 0$. A value of 0 indicates chance agreement only, while a value of 1.0 would indicate perfect agreement. A negative $\kappa$ value would indicate systematic disagreement between observers. It is generally accepted that a $\kappa$ value of 0.75 or higher reflects excellent agreement, $\kappa$ of 0.4 to 0.75 fair to good agreement and $\kappa$ values of less than 0.4 poor agreement.

The participants were allowed to grade their responses into two degrees of confidence. Grade I indicated a high degree of confidence, whereas grade II indicated doubt as to the precise classification of the tumour. To some extent the reason for this decision was the nature of the biopsy material itself; the amount of tumour tissue present in some of the biopsy specimens was so small or so badly traumatised that accurate classification was considered inappropriate, if not impossible.

\section{Results}

The results of this study are shown in tables 2 and 3 . The amount of agreement expressed as $\kappa$ values for each histological type of carcinoma is shown both overall and for grade I confidence. The figures in brackets represent the equivalent results from our previous study 
Table 3 Subclassification of non-small cell carcinoma: grade I confidence

\begin{tabular}{lll}
\hline Type & $\kappa$ value & $\begin{array}{l}\text { Value in } \\
\text { previous work }\end{array}$ \\
\hline Squamous cell & 0.73 & $(0.37)$ \\
Adenocarcinoma & 0.83 & $(0.58)$ \\
Large cell undifferentiated & 0.51 & $(0.22)$ \\
& 0.71 & $(0.36)$ \\
Overall & $(\mathrm{SE}=0.027)$ & \\
\hline
\end{tabular}

Table 4 Differentiation types of the 100 tumours: majority diagnosis

\begin{tabular}{lcc}
\hline Tumour type & Overall & Grade I confidence \\
\hline Squamous & 68 & 54 \\
Adenocarcinoma & 10 & 6 \\
Large cell undifferentiated & 22 & 0 \\
& 100 & 60 \\
\hline
\end{tabular}

which was published earlier in this Journal. ${ }^{2}$ The inclusion of a mucin stain in the current study has obviously made a significant difference to the consistency of histological categorisation. In our previous study where no such stain was provided, it became clear that less well differentiated carcinomas could not be accurately categorised because the current WHO classification includes undifferentiated tumours which produce mucin in the adenocarcinoma group. Therefore, any carcinoma that was not clearly squamous or clearly glandular in type could not be confidently categorised. This is reflected in the poor $\kappa$ values obtained in that paper. This problem is overcome by the inclusion of a mucin stain and has resulted in much improved $\kappa$ values.

It is clear from the results that cases were reported with grade I confidence either when the tumour was well differentiated, either squamous or adenocarcinoma, or when the biopsy was adequate enough to assess properly whether a less well differentiated tumour was producing mucin or not.

Kappa values of 0.73 and 0.83 for squamous carcinoma and adenocarcinoma, respectively, are very good, indicating that these diagnoses may be made with confidence on reasonable biopsy material. Even the diagnosis of large cell undifferentiated carcinoma, which is essentially a diagnosis of exclusion - that is, not squamous, not glandular and no mucin present, was made with reasonable consistency $(\kappa=0.51)$ with grade I confidence.

Overall, an average of $58 \%$ of the 100 cases were diagnosed with grade I confidence. Table 4 shows the differentiation type of the 100 cases based on the majority diagnosis both overall (100 cases) and where the majority of the pathologists gave a grade I confidence diagnosis (60 cases). This presumably reflects the relative frequency of the various types in Scotland. It is noteworthy (table 4) that while large cell undifferentiated carcinoma was diagnosed with grade I confidence on many occasions by individual pathologists, in not one case was this a majority view, suggesting that even with the mucin stain provided this is not a "robust" diagnosis on biopsy material.

\section{Discussion}

In Europe and North America, lung carcinoma kills more people than carcinoma of any other organ. Bronchial biopsy is an efficient way for the clinician to achieve a tissue diagnosis. Apart from providing a firm diagnosis, the pathologist will be asked to indicate whether the tumour is of small cell or non-small cell type, because there are important therapeutic implications for such a distinction. We have previously shown ${ }^{2}$ that histopathologists are extremely good at differentiating between small and nonsmall cell carcinoma on such material. Although at the present time, there is little therapeutic implication for the sub-division of nonsmall cell carcinoma types, we have shown that given adequate material for examination, the better differentiated tumours can be confidently sub-classified. Our study is not directly comparable with that of Thomas et al where they compared their biopsy results with full histological assessment from the resection specimens. We do however agree with their main conclusion that many non-small cell carcinomas should not be categorised further on limited material, especially as therapeutic implications are minimal.

The results of the current study are considerably better than those of our previous attempt. ${ }^{2}$ In part this was due to the inclusion of a mucin stain. It is arguable, however, how much the experience gained in reporting 100 previous cases under these conditions had improved our performance. In our previous studies ${ }^{67}$ we had noticed improved consistency in later batches of the same study, but in these previous studies, discussion of diagnostic criteria during our frequent meetings may have had some influence. Both in this and in the previous bronchial study we have used strict WHO criteria, thus permitting very little room for diagnostic manoeuvring. Also the $\kappa$ values, although displayed here as equivalents, should not be directly compared between this study and the previous one, because in the previous study a large category of small cell carcinoma was also included. It is noteworthy in this respect that in this study three pathologists made diagnoses of small cell carcinoma (all on the same section) and one pathologist made a diagnosis of small cell carcinoma on three separate occasions, even though none of these sections had been originally reported as small cell cancer.

Using the WHO classification, it is clear that squamous carcinoma and well differentiated (that is, glandular) adenocarcinoma are well categorised and we regard almost all other non-small cell carcinomas to be tumours which have not achieved sufficient differentiation to be included in one of the three better characterised groups (that is, squamous, adenocarcinoma and small cell carcinoma). We prefer, however, the original WHO classification $(1961)^{8}$ which placed large cell carcinoma with mucin production into the large cell anaplastic group rather than into the adenocarcinoma group, as this is more in keeping with current views of the histogenesis of large cell anaplastic carcinoma ${ }^{9}$ 
and in addition, from a purely practical point of view, would eliminate the necessity for a routine mucin stain. It was also clear from our two studies that the WHO criteria for squamous carcinoma are difficult to apply to bronchial biopsy specimens and it is likely that many tumours diagnosed as large cell anaplastic by our group would, if more tissue was available, show either keratin production or the presence of intercellular bridges ("prickles"), although the latter feature did seem to cause particular diagnostic difficulty among the group.

The 100 tumour positive cases used in this study were pulled at random from the pathology files of 10 different laboratories. The presence of carcinoma was confirmed in all of them. This suggests that false positive reporting of lung carcinoma on bronchial biopsy material is not a major problem for histopathologists. We further conclude that a diagnosis of non-small cell carcinoma-not otherwise specified-is a respectable diagnosis to be made on such material. However, if the tumour is well enough differentiated and present in sufficient amount for a confident diagnosis of a specific sub-type to be made, then this can be done consistently by competent histopathologists.
The Scottish Panel for the Consistency of Histopathological Reporting was set up by Professor J Swanson Beck in 1987. This study was funded by the Clinical Resource and Audit Group of the Scottish Office Home and Health Department and by Tayside Health Board. We are grateful to Mrs Joyce Duncan and Miss Margaret Brough for administrative and secretarial assistance.

1 World Health Organisation. Histological typing of lung tumours. In: International histological classification of tumour No. 1. 2nd edn. Geneva: World Health Organisation, 1981.

2 Burnett RA, Beck JS, Howatson SR, Lee FD, Lessells AM, McLaren KM, et al. Observer variability in histopathological reporting of malignant bronchial biopsy specimens. f Clin Pathol 1994;47:711-13.

3 Thomas J St J, Lamb D, Ashcroft T, Corrin B, Edwards CW, et al. How reliable is the diagnosis of lung cancer using small biopsy specimens? Report of a UK CCCR lung cancer working party. Thorax 1993;48:1135-9.

4 Willis RA. Epithelial tumours of the trachea, bronchi and lung. In: Pathology of tumours. 3rd edn. London: Butterworths, 1960:369.

5 Roggli VL, Vollmer RT, Greenberg SD, McGavron MH, Spjut HJ, Yesner R. Lung cancer heterogeneity: A blinded randomized study of 100 consecutive cases. Hum Pathol 1985;16:569-78.

6 Robertson AJ, Anderson JM, Beck JS, Burnett RA, Howatson SR, Lee FD, et al. Observer variability in histopathological reporting of cervical biopsy specimens. $\mathcal{F}$ Clin Pathol 1989; 42:231-8.

7 Robertson AJ, Beck JS, Burnett RA, Howatson SR, Lee $\mathrm{FD}$, Lessells AM, et al. Observer variability in histological reporting of transitional cell carcinoma and epithelial dysplasia in bladders. $\mathcal{F}$ Clin Pathol 1990;43:17-21.

8 World Health Organisation. Histological typing of lung tumours. In: International histological classification of tumours. No. 1. 1st edn. Geneva: World Health Organisation, 1961.

9 Chuang MT, Marchevsky A, Teirstein A, Kirschner PA, Kleinerman J. The diagnosis of lung cancer by fibreoptic bronchoscopy. I. Problems in the histologic classification of non-small cell carcinomas. Thorax 1984;39:175-8. 